\title{
Heat Capacity of Gaseous Hexafluoroethane ${ }^{1}$
}

\section{John S. Wicklund, Howard W. Flieger, Jr., and Joseph F. Masi}

An accurate flow calorimeter has been used to measure the heat capacity $\left(C_{p}\right)$ of gaseous hexafluoroethane $\left(\mathrm{C}_{2} \mathrm{~F}_{6}\right)$ at $-50^{\circ},-20^{\circ},+10^{\circ},+50^{\circ}$, and $+90^{\circ} \mathrm{C}$ and at $0.5-, 1.0-$, and 1.5 -atmosphere pressure. The results are believed to be accurate to \pm 0.1 percent.

The values of $C_{p}$ have been extrapolated to zero pressure at each temperature; the ideal-gas heat capacities thus obtained at the five temperatures are, respectively, 20.99, 22.79, 24.43, 26.45 , and 28.24 calories mole $^{-1}$ degree $^{-1}$. Calculated results, using recent frequency assignments and molecular data, were 0.4 to 0.7 percent higher than the experimental results.

The values of the pressure coefficient of heat capacity at the five temperatures have been used in conjunction with literature data to obtain an equation of state for hexafluoroethane at low pressures.

\section{Introduction}

Accurate measurements of gas heat capacity over a range of low pressures and at several temperatures provide important information about both the ideal gas and the low-pressure equation of state. The flow calorimeter used in this investigation has been previously described., ${ }^{2} 3$ This measures the heat capacity of gases with an accuracy of the order of 0.1 percent, from $-50^{\circ}$ to $+100^{\circ} \mathrm{C}$, and up to about $1.5 \mathrm{~atm}$.

The heat capacity of gaseous hexafluoroethane is of particular interest because of the question of the size of the potential barrier to internal rotation. Pace and Aston ${ }^{4}$ made calorimetric measurements on the solid and liquid, and obtained third-law entropies of the ideal gas; comparison with certain spectroscopic calculations led them to a value of 4,350 calories mole $^{-1}$ for the potential barrier.

\section{Experimental Procedure}

\section{1. Material}

A specially purified sample of $\mathrm{C}_{2} \mathrm{~F}_{6}$ was obtained from Minnesota Mining \& Manufacturing Corp. It was further purified by evaporation at the temperature of a slush of dry ice in a 50-50 mixture of $\mathrm{CHCl}_{3}$ and $\mathrm{CCl}_{4}$, and subsequent condensation in a trap surrounded by liquid nitrogen, while pumping with a high-vacuum apparatus. Approximately $385 \mathrm{~g}$ of final sample were obtained in this manner.

A mass-spectrographic analysis of the sample as used indicated a purity of 99.4 mole percent or better, with a maximum of 0.1 mole percent $\mathrm{CO}_{2}$, 0.5 percent $\mathrm{N}_{2}$, and/or CO, and a trace of a hydrocarbon. A separate test with a phosphoric acid conductimetric method indicated only $0.02 \mathrm{mg}$ of water per liter of sample.

\subsection{Apparatus and Method}

The construction of the flow calorimeter and its operation have been described in detail elsewhere (footnote 2); a brief résumé has also been published (footnote 3 ).

A new electronic control system, designed and built at the National Bureau of Standards, was used

This work was supported by the Ordnance Corps, Department of the Army. 2 J. F. Masi and B. Petkof, J. Research NBS 48, 179 (1952). RP2303.

3 J. F. Masi, J. Am. Chem. Soc. 74, 4738 (1952).

E. L. Pace and J. G. Aston, J. Am. Chem. Soc. 70, 566 (1949) successfully to control the temperature of the radiation shield to within $\pm 0.01 \mathrm{deg} \mathrm{C}$ of the average temperature of the tube containing the heated gas. This control is to be described in a future publication.

The heat capacity was determined at a number of different flow rates at each temperature and pressure, so that any residual heat leak could be eliminated by extrapolation to the zero of reciprocal rate. "Blank" determinations were made at several rates at each temperature and pressure to measure the amount of cooling, $\delta T$, experienced by the gas in passing through the calorimeter when no heat was applied. A total of 67 heat-capacity experiments and 75 blank experiments were made.

\section{Results}

\section{1. Heat Capacity of Hexafluoroethane}

If $W$ is the power supplied to the heater, $\mathrm{F}$ is the rate of flow of gas, $\Delta T$ is the observed rise in temperature, and $\delta T$ is the fall in temperature in a corresponding blank experiment, the apparent heat capacity is calculated by

$$
C=\frac{W F^{-1}}{\Delta T+\delta T}
$$

The values of $C$ were corrected for the deviation of the observed mean pressure and mean temperature from the nominal values. These corrections were small in all cases. The corrected heat capacity, called $C_{p}$ (observed), was plotted against the reciprocal of the rate of flow at each temperature and pressure and straight lines were fitted to the points by the method of least squares.

The least-square lines had very small slopes; moreover, the slopes were not constant with pressure at some of the temperatures. An analysis of variance was performed to test the significance of the slopes obtained. As a result, it was decided to abandon, in this case, the usual linear extrapolation to infinite rate, and to obtain the final values of heat capacity by simple averaging of the $C_{p}$ (observed) at each temperature and pressure. The results of this process are listed in table 1, converted to calories mole- ${ }^{-1}$ degree $^{-1}$. (The calorie is $4.1840 \mathrm{j}$, the molecular weight is 138.02 , and $0^{\circ} \mathrm{C}=273.16^{\circ} \mathrm{K}$ ). Each of the experimental values listed in table 1 is the mean of at least four determinations. Following each tabular value is the standard deviation of the experiments from the mean; the average of these deviations is 


\begin{tabular}{|c|c|c|c|c|c|}
\hline \multirow[t]{2}{*}{ Temperature, ${ }^{\circ} \mathrm{C} \ldots$} & -50.00 & -20.00 & 10.00 & 50.00 & 90.00 \\
\hline & \multicolumn{5}{|c|}{$C_{p}$, calories mole ${ }^{-1}$ degree $^{-1}$} \\
\hline $\begin{array}{l}\text { Pressure: } \\
1.50 \mathrm{~atm} \\
1.00 \mathrm{~atm} \\
0.50 \mathrm{~atm} \\
C_{p}{ }_{p}, \text { observed } \\
C_{p}{ }^{0} \text {, spectroscopic } \\
\Delta C_{p} / \Delta P \text {, cal mole }{ }^{-1} \mathrm{deg}^{-1} \mathrm{~atm}^{-1}\end{array}$ & $\begin{array}{l}21.803, \pm 0.005 \\
21.539, \pm 0.009 \\
21.257, \pm 0.010 \\
20.99 \\
21.13 \\
0.546\end{array}$ & $\begin{array}{l}23.234, \pm 0.003 \\
23.072, \pm 0.007 \\
\text { 22. } 938, \pm 0.009 \\
\text { 22. } 79 \\
\text { 22. } 90 \\
\quad 0.296\end{array}$ & $\begin{array}{l}\text { 24. } 718, \pm 0.011 \\
24.617, \pm 0.009 \\
\text { 24. } 526, \pm 0.010 \\
\text { 24. } 43 \\
\text { 24. } 56 \\
\quad 0.192\end{array}$ & $\begin{array}{l}26.615, \pm 0.009 \\
26.547, \pm 0.016 \\
26.506, \pm 0.006 \\
26.45 \\
26.56 \\
\quad 0.109\end{array}$ & $\begin{array}{l}28.367, \pm 0.011 \\
28.333, \pm 0.008 \\
28.283, \pm 0.019 \\
28.24 \\
28.36 \\
\quad 0.084\end{array}$ \\
\hline
\end{tabular}

\pm 0.037 percent compared with \pm 0.036 percent for the corresponding precision index in the case of leastsquare lines fitted to the experiments.

The final values of heat capacity at finite pressures in table 1 were extrapolated linearly to zero pressure at each temperature, and the results are given as " $C_{p}{ }^{0}$, observed." It is believed that these heatcapacity values for the ideal gas $\mathrm{C}_{2} \mathrm{~F}_{6}$ are reliable to better than \pm 0.15 percent.

An assignment of the fundamental frequencies of $\mathrm{C}_{2} \mathrm{~F}_{6}$ was made by Nielsen, Richards, and McMurry. ${ }^{5}$ Pace and Aston (footnote 4) have used this assignment in conjunction with their experimental thirdlaw entropies at $176.61^{\circ}$ and $194.87^{\circ} \mathrm{K}$ to obtain a value of 4,350 calories mole ${ }^{-1}$ for the barrier to internal rotation. A recent note by Mann and Plyler ${ }^{6}$ puts the lowest frequency $\left(\nu_{9}\right)$ at $220 \mathrm{~cm}^{-1}$ by direct observation in the infrared, and suggests new estimates for the interatomic distances. The barrier energy becomes 3,920 cal on the basis of these changes. The heat capacity has been calculated from this slightly revised assignment, for the harmonic-oscillator, rigid-rotator approximation, and the results at the temperatures of the present experiments are given in table 1 as " $C_{p}{ }^{0}$, spectroscopic." They are seen to be 0.4 to 0.7 percent higher than the observed values.

\subsection{Equation of State of Hexafluoroethane}

It is assumed here that in the low-pressure region covered by these experiments the equation of state of $\mathrm{C}_{2} \mathrm{~F}_{6}$ is of the form

$$
P V=R T+B P \text {, }
$$

where $B$ is a function of the temperature. The thermodynamic relation

$$
\left(\frac{\partial C_{p}}{\partial P}\right)_{T}=-T\left(\frac{\partial^{2} B}{\partial T^{2}}\right)_{P}
$$

must be satisfied by the second derivative of the second virial coefficient $B$. The values of $\Delta C_{p} / \Delta P$ listed in the last line of table 1 were taken to be the lefthand member of eq (3), and B was assigned the form proposed by Hirschfelder, McClure, and Weeks: ${ }^{7}$

$$
B=b-c e^{a T} .
$$

The values of $c$ and $a$ were then chosen to fit the data of this experiment, whereas $b$ was obtained from the vapor-density data of Pace and Aston (see footnote 4 ) at $25^{\circ} \mathrm{C}$. The final expression for

5 J. R. Nielsen, C. M. Richards, and H. L. McMurry, J. Chem. Phys. 16, 67 (1948).

6 D. E. Mann and E. K. Plyler. J. Chem. Phys. 21, 1116 (1953).

${ }^{7}$ J. O. Hirschfelder, E. T. McClure, and I. F. Weeks, J. Chem. Phys. 10, the virial coefficient in eq (2) was

$$
B=91-58.2 e^{495 / T} \mathrm{~cm}^{3} \mathrm{~mole}^{-1} \text {. }
$$

The experimental values of $\Delta C_{p} / \Delta P$ are plotted as as circles in figure 1 . The calculated values obtained by differentiating eq (5) are indicated by the solid curve, whereas the dashed curve is calculated from the Berthelot equation, using the critical constants given by Pace and Aston (see footnote 4). Table 2 shows that eq (5) reprorluces the results of Pace and Aston's calculation of $B$ from vapor pressure and heat of vaporization measurements, at least as well

\begin{tabular}{|c|c|c|c|}
\hline \multirow[b]{2}{*}{$\begin{array}{l}\text { Tempera- } \\
\text { ture, } T\end{array}$} & \multicolumn{3}{|c|}{ Value of $B$} \\
\hline & $\begin{array}{l}\text { Data of Pace } \\
\text { and Aston } \\
\text { (see foot: } \\
\text { note } 4 \text { ) }\end{array}$ & $\begin{array}{l}\text { Berthelot } \\
\text { equation }\end{array}$ & Equation (5) \\
\hline $\begin{array}{c}{ }^{\circ} K \\
179.96 \\
180.22 \\
188.30 \\
190.05 \\
194.87 \\
194.90 \\
195.10 \\
195.21 \\
298.16\end{array}$ & $\begin{array}{l}c m^{3} \text { mole-1 } \\
-837 \\
-815 \\
-758 \\
-766 \\
-641 \\
-653 \\
-611 \\
-624 \\
-215 \text { (avg) }\end{array}$ & $\begin{array}{c}c m^{3} \text { mole-1 } \\
-822 \\
-819 \\
-738 \\
-721 \\
-678 \\
-678 \\
-676 \\
-675 \\
-268\end{array}$ & $\begin{array}{c}c m^{3} \text { mole-1 } \\
-820 \\
-816 \\
-715 \\
-696 \\
-647 \\
-647 \\
-646 \\
-644 \\
(-215)\end{array}$ \\
\hline
\end{tabular}
as the Berthelot equation.

TABLE 2. Comparison between equations of state of gaseous hexafluoroethane

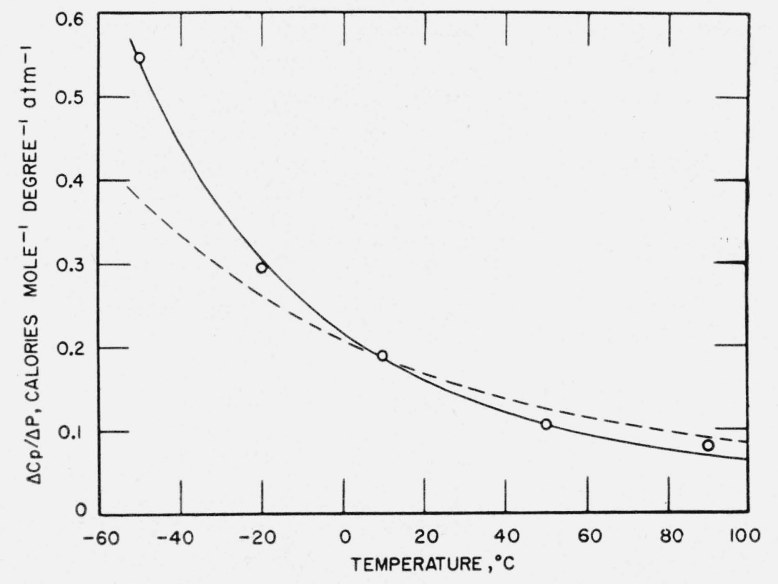

Figure 1. Pressure coefficient of heat capacity of hexafluoroethane.

, this research; — equation.

Washington, May 7, 1953. 